\title{
Service Blueprinting and Business Process Modeling Notation (BPMN): A Conceptual Comparison
}

\author{
Yahya Kazemzadeh $^{1}$, Simon K. Milton ${ }^{1} \&$ Lester W. Johnson ${ }^{2}$ \\ ${ }^{1}$ Department of Computing and Information Systems, The University of Melbourne, Melbourne, Australia \\ ${ }^{2}$ Swinburne Business School, Melbourne, Australia \\ Correspondence: Yahya Kazemzadeh, Department of Computing and Information Systems, The University of \\ Melbourne, Parkville, VIC 3010, Australia. E-mail: yahyak@student.unimelb.edu.au
}

$\begin{array}{lr}\text { Received: January 10, } 2015 & \text { Accepted: February 27, } 2015 \quad \text { Online Published: April 30, } 2015 \\ \text { doi:10.5539/ass.v11n12p307 } & \text { URL: http://dx.doi.org/10.5539/ass.v11n12p307 }\end{array}$

\begin{abstract}
The modeling of business processes has become a central aspect in how businesses understand, support, and communicate about their processes. Two prominent approaches are service blueprinting and business process modeling notation (BPMN). Service blueprinting supports customer service processes whilst BPMN helps understand a firm's processes with particular focus on how information and communications technology supports processes, and also for process automation. To fully support services through an organization's processes, there needs to be a complete understanding of how these two process representations relate. Hitherto only a partial comparison has been undertaken by Milton and Johnson in 2012. Therefore we ask the question, what are the specific similarities and differences between these two approaches? To answer this question, we employed the method of conceptual evaluation to conduct a two-way conceptual comparison of service blueprinting and BPMN. We found specific similarities and differences between the two modeling approaches. Understanding how to represent service blueprint concepts in BPMN is important for supporting service-processes with information technology and for automating aspects of those processes. Furthermore, knowing the limitations of how service blueprints support BPMN means that mapping internal processes to service processes can be done with minimal loss in semantics.
\end{abstract}

Keywords: service blueprinting, business process modeling notation (BPMN), concept, conceptual evaluation, conceptual comparison

\section{Introduction}

Service blueprinting and the business process modeling notation (BPMN) are process modeling techniques with different perspectives toward service processes and operations. Service blueprinting is a domain specific modeling approach (for service processes), designed by service marketers to address challenges and difficulties regarding the interaction of the customer with the service provider. Service blueprints are easy to understand and all stakeholders (customers, organization's employees and managers) can communicate with them (Bitner, Ostrom, \& Morgan, 2008). BPMN is a general purpose modeling approach (for any process), used by several companies to analyze and document their business processes. We aim in this study to compare these models, and to understand their conceptual similarities and differences in more detail. The main research question is:

What are the conceptual similarities and differences of service blueprinting and BPMN?

Milton and Johnson (2012) employed a similar research method to understand how well service blueprinting concepts are supported by BPMN concepts. However, this study not only answers this question in more detail, it is bi-directional in that it assesses how well BPMN concepts are supported by service blueprinting concepts rather than just seeing how well BPMN supports service blueprinting. Specifically, this study aims to answer two subordinate research questions: 1) how well does BPMN conceptually support service blueprinting? And 2) how well does service blueprinting conceptually support BPMN? Answering these two questions clarifies the conceptual similarities and differences of service blueprinting and BPMN. We (in press, 2015a, 2015c) successfully used the method of conceptual evaluation to compare various modeling formalisms and there is no alternative to this method for conducting a comparison of this nature. Considering that there is a growing interest in BPMN (Chinosi \& Trombetta, 2012), the findings of this comparison can be used to refine BPMN to meet a 
customer's perspective as well as an organizational perspective and it can provide a foundation to understand how they can be used together.

This paper continues with a comprehensive explanation of research design and method. Following this, service blueprinting and BPMN are explained precisely. We then present a conceptual evaluation of BPMN against service blueprinting for the purpose of answering the first research sub-question. The next part is dedicated to the conceptual evaluation of service blueprinting against BPMN in order to answer the second research sub-question. Finally, we consolidate findings and conclude.

\section{Research Design}

This section explains an improved version of the method of conceptual evaluation (Milton \& Johnson, 2012) in detail. The purpose of this method is to conceptually compare a pair of process modeling formalisms. Considering A and B as the process modeling formalisms under study, the method has five steps. The first two steps determine concepts of A and B. Then step 3 explains how to compare the concepts of B using the concepts of $\mathrm{A}$ as a kind of yard stick or benchmark. Step 3 has two dimensions: presenting the results of comparison in a tabular form and then explaining the nature of the gaps between concepts. Step 4 resembles the third and includes the comparison of concepts of A with the concepts of B and reverses the roles of the formalisms compared with step 3. Step 5 reports the findings and implications from the results of steps 3 and 4 . The details of steps are as follows:

Step 1. Determine the concepts of A. The concepts for A are $<\mathrm{a} 1>,<\mathrm{a} 2>, \ldots,<\mathrm{an}>$.

Step 2. Determine the concepts of B. The concepts of B are $<\mathrm{b} 1>,<\mathrm{b} 2>, \ldots,<\mathrm{bm}>$.

Step 3. Perform a conceptual evaluation of B against A. This means each concept from A will be compared with concepts from B. We utilize semiotic theories for this purpose. Milton and Kazmierczak (2004, p.30) state two reasons for employing semiotic theories to compare concepts with each other:

"Firstly, terms and concepts are clearly semiotically related. Secondly, comparison of concepts is semantic with semiotic theory providing an ideal basis for explaining semantic differences in terms."

Specifically, concepts "span parts of a semantic field (Eco, 1976), or conceptual plane (Cruse, 2000; Culler, 1976). Alternatively, each term possesses an essential depth (Liska, 1996) which similarly evokes the conceptual span of a term" (Milton \& Kazmierczak, 2004, p.30). In fact, when we compare two concepts from two different process modeling formalisms, we compare the similarities and differences of the "semantic field" associated with each concept.

We define three categories of the results when we compare $<a \mathrm{a}>(\mathrm{i}=1 \ldots \mathrm{n})$ with concepts from B: full respective coverage of the semantic field $(\checkmark)$, partial respective coverage of the semantic field $(\checkmark p)$ and no coverage of the semantic field ( $\mathbf{x})$. Full coverage happens when the semantic field of $<$ ai $>$ has total overlap with the semantic field of one or a combination of more than one concepts from B. Partial coverage happens when the semantic field of $<$ ai $>$ has partial overlap with the semantic field of one or a combination of more than one concepts from B. No coverage occurs when we cannot find any overlap between the semantic field of $<\mathrm{ai}>$ with the semantic field of one concept or any combination of concepts from B. We first investigate to see if there is a total overlap between the semantic field of $<\mathrm{ai}>$ and the concepts from B. If not, we search for partial overlap.

It is important to know that we always choose a simple concept or a combination of concepts from B that includes a minimum number of concepts still maintaining maximum coverage. This simple or combination of concepts is called "supportive concept". Specifically, $<\mathrm{bi}^{\prime}>, \ldots,<\mathrm{bj}^{\prime}>\left(0<\mathrm{i}^{\prime} \leq \mathrm{j}\right.$ ' $\left.\leq \mathrm{m}\right)$ are the concepts from B, altogether being a supportive concept, in which the combination of semantic fields fully or partially covers $<\mathrm{ai}>$. The supportive concept is represented as $\left\langle\mathrm{bi}^{\prime}+\ldots+\mathrm{bj}^{\prime}\right\rangle$.

However, more generally, there may be cases where the same semantic field, $<\mathrm{ai}>$, is partly or fully covered by different distinct compound concepts from $\mathrm{B}$. Specifically, $\mathrm{Si}(\mathrm{B})$ is a group of compound concepts, each member of which independently covers $<\mathrm{ai}>$ (either fully or partially). Then $\operatorname{Si}(\mathrm{B})=\left\{<\mathrm{bi}^{\prime}+\ldots+\mathrm{bj}^{\prime}>, \ldots,<\mathrm{bi}+\ldots+\right.$ $\left.\mathrm{bj}^{\prime \prime}>\right\}\left(0<\mathrm{i}^{\prime} \leq \mathrm{j}^{\prime} \leq \mathrm{i}^{\prime \prime} \leq \mathrm{j}^{\prime \prime} \leq \mathrm{m}\right)$. This specifically, means that $<\mathrm{ai}>$ is covered by $<\mathrm{bi}^{\prime}+\ldots+$ bj'> but also $<\mathrm{ai}>$ is independently covered by $<\mathrm{bi}^{\prime \prime}+\ldots+$ bj" $>$. However, in practice usually we cannot find more than one supportive concept. If there are no concepts in $\mathrm{B}$ to cover the semantic field of $<\mathrm{ai}>$ at all, then $\operatorname{Si}(\mathrm{B})=\{\varnothing\}$. Figure 1 pictorially represents the explained three categories of results for the coverage of $<$ ai $>$ by $\mathrm{Si}(\mathrm{B})$.

We present the results in a tabular form and then discuss the nature of the gaps utilizing semiotic theories according to the represented order of concepts of A. Table 1 presents an example of a table which is used to represent the results of comparing the concepts of A with the concepts of B. 
Table 1. Example-Results of conceptual evaluation of B against A

\begin{tabular}{ccc}
\hline Concepts of A & Degree of overlap & Supportive concepts of B \\
\hline$<\mathrm{a} 1>$ & $\checkmark / \checkmark \mathrm{p} / \mathbf{x}$ & $\mathrm{S} 1(\mathrm{~B})$ \\
$\ldots$ & $\ldots$ & $\ldots$ \\
$<$ an $>$ & $\checkmark / \checkmark \mathrm{p} / \boldsymbol{x}$ & $\mathrm{Sn}(\mathrm{B})$ \\
\hline
\end{tabular}
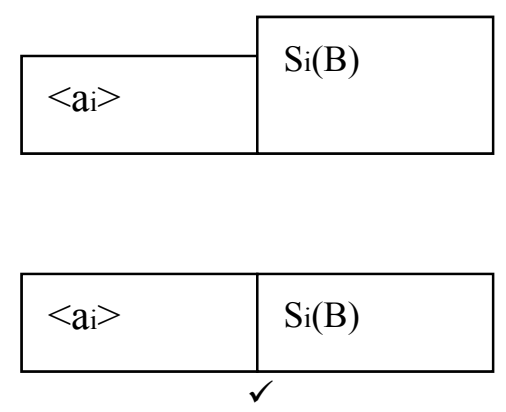
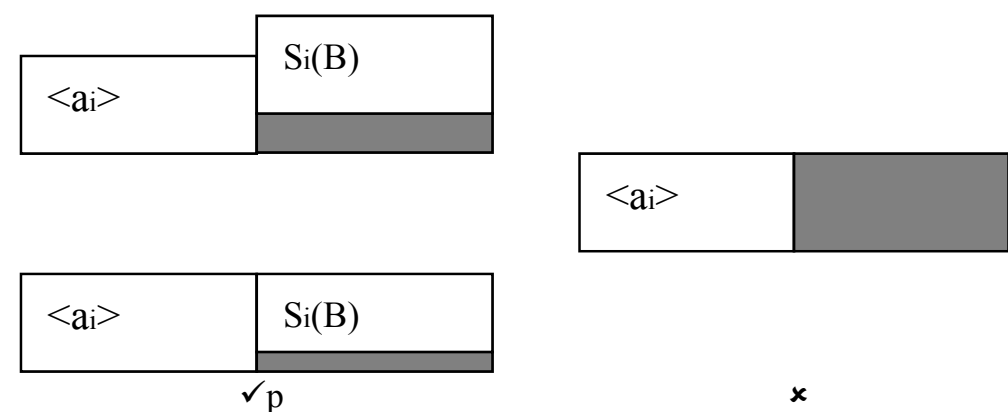

Figure 1. Degree of overlap in coverage of semantic field for $<$ ai $>$ by $\operatorname{Si}(B)$

While we used "+" to present a compound concept, the word "plus" is used during discussions for this purpose. The terms "fully covers", "completely covers", "fully supports" and "completely supports" are used to describe full coverage of $<$ ai $>$ with supportive concepts from B. For partial coverage, the terms "partially covers", "partly covers", "partially supports" and "partly supports" are used.

Step 4. This step resembles Step 3, dedicated to a conceptual evaluation of A against B.

Step 5. Consolidate the findings and explain the implications from the results of steps 3 and 4 . Two major findings in this step can be:

a) Suppose $<$ bi $^{\prime}+\ldots+$ bj' $^{\prime}>$ fully covers $<$ ai $>, \ldots,<$ aj $>$ and $<$ ai $+\ldots+$ aj $>$ fully covers $\left\langle\right.$ bi $^{\prime}>, \ldots,<$ bj' $^{\prime}>$. Then: $<b^{\prime}+\ldots+b^{\prime}>$ covers the same semantic field as $<a i+\ldots+$ aj $>$.

b) Suppose $<\mathrm{bi}^{\prime}+\ldots+\mathrm{bj}^{\prime}>$ fully covers $<$ ai $>, \ldots,<\mathrm{aj}>$ and $<\mathrm{ai}+\ldots+$ aj $>$ partially covers at least one concept from $\left\langle\mathrm{bi}^{\prime}>, \ldots,<\mathrm{bj}^{\prime}\right\rangle$. Then: $\left\langle\mathrm{bi}^{\prime}+\ldots+\mathrm{bj}^{\prime}>\right.$ has a broader semantic field than $<a \mathrm{ai}+\ldots+$ aj $>$.

\section{Service Blueprinting}

Service blueprinting has a long history of service marketing and innovation and is used in understanding existing services or planning new ones. Originally proposed by Shostack (1982, 1984, 1987), Kingman-Brundage (1989, 1991, 1993, 1995) further developed it, calling it service mapping.

A service blueprint examines customer interactions with a service company including interaction with individuals or technologies (e.g., websites), and is best created through cross-functional teams and customers (Bitner et al., 2008). All contact points, support processes, physical props / design and other process functions are represented to understand a crucial point of difference between firms: customer experience (Alonzo-Helton, Fletcher-Brown, \& Stephens, 2013) in service delivery (Ojasalo, 2012). Service blueprinting is a structured way to help manage customer experience and also to reach customer and firm goals (Bitner et al., 2008).

There are two dimensions in a blueprint: "the horizontal axis represents the chronology of actions conducted by the service customer and service provider. The vertical axis distinguishes between different areas of actions. These areas of actions are separated by different 'lines:" (Fließ \& Kleinaltenkamp, 2004, p. 396). Figure 2 presents a service blueprint for a hotel stay.

In Figure 2, actor actions and physical evidences are shown by boxes. Sequences of boxes show action flow for each actor. Arrows between actor actions show communication flow (Milton \& Johnson, 2012). The horizontal segmentations of actions represent actor categories. The actor categories are customer, onstage employees, backstage employees (or systems), support and management. Four lines segregate actors based on distance from, and interaction with, the customer and were in the original conception of service blueprints (Bitner et al., 2008) and have been augmented by: the lines of interaction, visibility, internal interaction, and implementation. A fifth, called the line of order penetration, was introduced by Fließ \& Kleinaltenkamp (2004) and lies between the line of internal interaction, and the line of implementation. 
The line of interaction separates customer actions from the actions of the service company's employees and its systems. These employees and systems are either visible to the customer or lie further away. The line of visibility separates those actions of which the customer is aware from others not visible but supporting the customer (and indeed from other actors deeper in the organization). The line of internal interaction separates backstage actions that directly support customers in the service encounter from other actions of the business. Those actors whose activities are induced by customer actions are those said to be 'directly supporting customers', whether visible or in the 'backstage' and "can be carried out after having been started by the customer" (Fließ \& Kleinaltenkamp, 2004, p. 396) or induced by other customer-related events external to the business. The line of order penetration separates customer-induced actions from actions that are independent of customers. Customer independent actions are "independent from a specific customer and only rely on the service company's internal production factors" (Fließ \& Kleinaltenkamp, 2004, p. 397). Finally, the line of implementation separates support actions from managerial actions.

The core concepts of service blueprinting are shown in Table 2, which were defined earlier, with their precise definitions to facilitate the conceptual comparison of service blueprinting with BPMN. The following section is dedicated to an introduction of business process modeling notation (BPMN).

Table 2. Concepts in service blueprinting

\begin{tabular}{ll}
\hline Concept & Definition \\
\hline$<$ Action $>$ & $\begin{array}{l}\text { Actions that customer, onstage personnel, backstage personnel (systems), } \\
\text { support and management perform in a service process (Milton \& Johnson, } \\
2012) .\end{array}$ \\
\hline$<$ Actor Categories $>$ & $\begin{array}{l}\text { Actor categories are customer, onstage personnel, backstage personnel } \\
\text { (systems), support and management (Bitner et al., 2008). }\end{array}$ \\
\hline$<$ Action Flow $>$ & $\begin{array}{l}\text { Action Flow presents the sequence of actions by an actor (Milton \& } \\
\text { Johnson, 2012). }\end{array}$ \\
\hline$<$ Communication Flow $>$ & $\begin{array}{l}\text { Communication Flow presents the flow of communication between any } \\
\text { actors in the service (Milton \& Johnson, 2012). }\end{array}$ \\
\hline$<$ Line of Interaction $>$ & $\begin{array}{l}\text { Line of Interaction is an interface between customer and frontline } \\
\text { employees (systems) (Bitner et al., 2008). }\end{array}$ \\
\hline$<$ Line of Visibility $>$ & $\begin{array}{l}\text { Line of Visibility is an interface between onstage and backstage } \\
\text { employees (systems) (Bitner et al., 2008). }\end{array}$ \\
\hline$<$ Line of Internal Interaction $>$ & $\begin{array}{l}\text { Line of Internal Interaction is an interface between backstage employees } \\
\text { (systems) and support employees (systems) (Bitner et al., 2008). }\end{array}$ \\
\hline$<$ Line of Order Penetration $>$ & $\begin{array}{l}\text { Line of Order Penetration is an interface between customer induced } \\
\text { actions and customer independent actions (Fließ \& Kleinaltenkamp, } \\
\text { 2004). }\end{array}$ \\
\hline$<$ Line of Implementation $>$ & $\begin{array}{l}\text { Line of Implementation is an interface between support employees } \\
\text { (systems) and managerial actions (Bitner et al., 2008). }\end{array}$ \\
\hline$<$ Props and Physical Evidence $>$ & $\begin{array}{l}\text { Props and Physical Evidence are all the tangibles that customers see } \\
\text { during the service process and influence the customer's perception of } \\
\text { quality (Bitner et al., 2008). }\end{array}$ \\
\hline
\end{tabular}

\section{Business Process Modeling Notation (BPMN)}

BPMN is an important process modeling technique and has attracted considerable research attention (Muehlen \& Recker 2008). BPMN was first published by a consortium of tool vendors (BPMI.org 2004) but later allowed the Object Management Group (OMG) to publish it (BPMI.org and OMG, 2006). We cover BPMN version 2.0 (OMG, 2011).

BPMN has a core set of constructs and an extended set. The core set is for business analysts and non-technical users to model processes and activities for all stakeholders (Ko, Lee, \& Lee, 2009; Recker, 2011). The extended set is for technical users allowing for more complexity and can be used for detailed design and automation in "workflow engineering, simulation, or web service composition" (Recker, 2010, p. 183). We consider the core 
set of BPMN constructs because we want to compare BPMN with service blueprinting, which is used to communicate with all stakeholders in a service process (including non-technical stakeholders). Figure 2 shows a BPMN diagram of the hotel stay example that we used in the service blueprinting section.

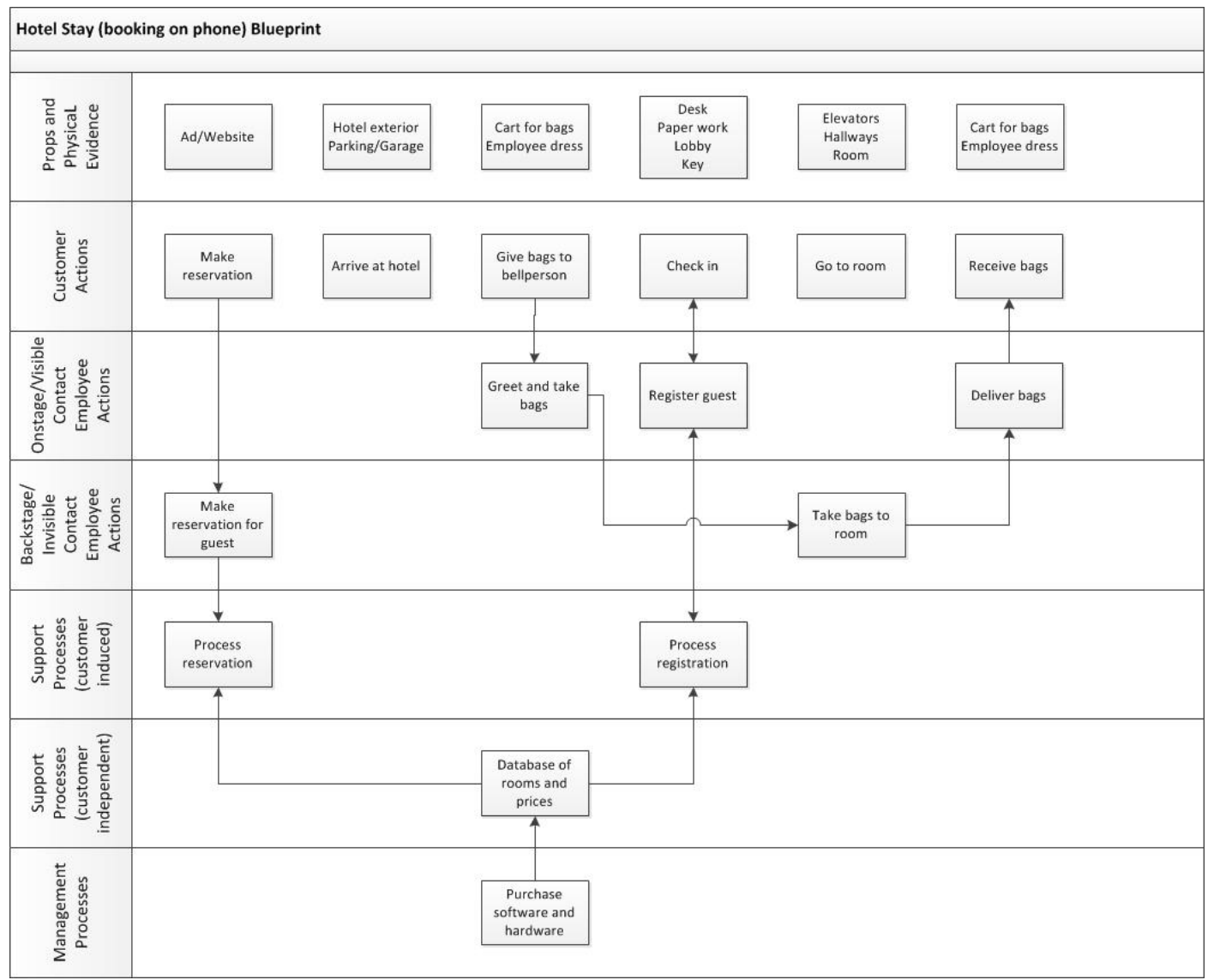

Figure 2. Service Blueprint example - Hotel Stay

There are four categories in BPMN basic constructs: flow objects, connecting objects, swimlanes and artefacts. Flow objects include event, activity and gateway, and connecting objects include sequence flow, message flow and association. Processes are separated into pools and lanes. Other artefacts are data object, group and text annotation.

An event triggering activity is shown in BPMN by a circle. There are three types of trigger: start event (when a participant begins a process), intermediate event (happens at the middle of a process) and end event (when a participant finishes a process). A super-type event happens at any time in a process and affects the flow (Kazemzadeh, Milton, \& Johnson, 2015b).

Activity denotes actions and other processing in processes, and can be atomic (a specific task that won't be broken down) or compound (has sub-process). Activities are shown with rounded rectangles. Complex activities are denoted using a plus sign in the bottom center of the activity rectangle.

A gateway allows divergence and convergence of process flows. OMG (2011) defines six basic types of splitting and joining process flows. All are shown as diamonds with markings inside the diamond differentiating between the types. This study defines a super-type gateway as one allowing branching, forking, merging, or joining of paths: "whether exactly one (exclusive "or"), more than one ("or"), or all ("and") of the activities entering or leaving the join or split, respectively, are required prior to (join) or must follow (split)" (Milton \& Johnson, 2012, p.612). In the hotel stay example the gateway determines branching of sequence flows: if the customer has bags then he give bags to a bellperson, otherwise he/she will proceed to check-in.

A sequence flow orders activities and is shown by a solid line with an arrow showing sequence of activities. A message flow, with attached label, shows a message flowing between activities and is denoted by a dashed line with a solid arrow, which indicates the direction of communication, the label showing the type of communication. 


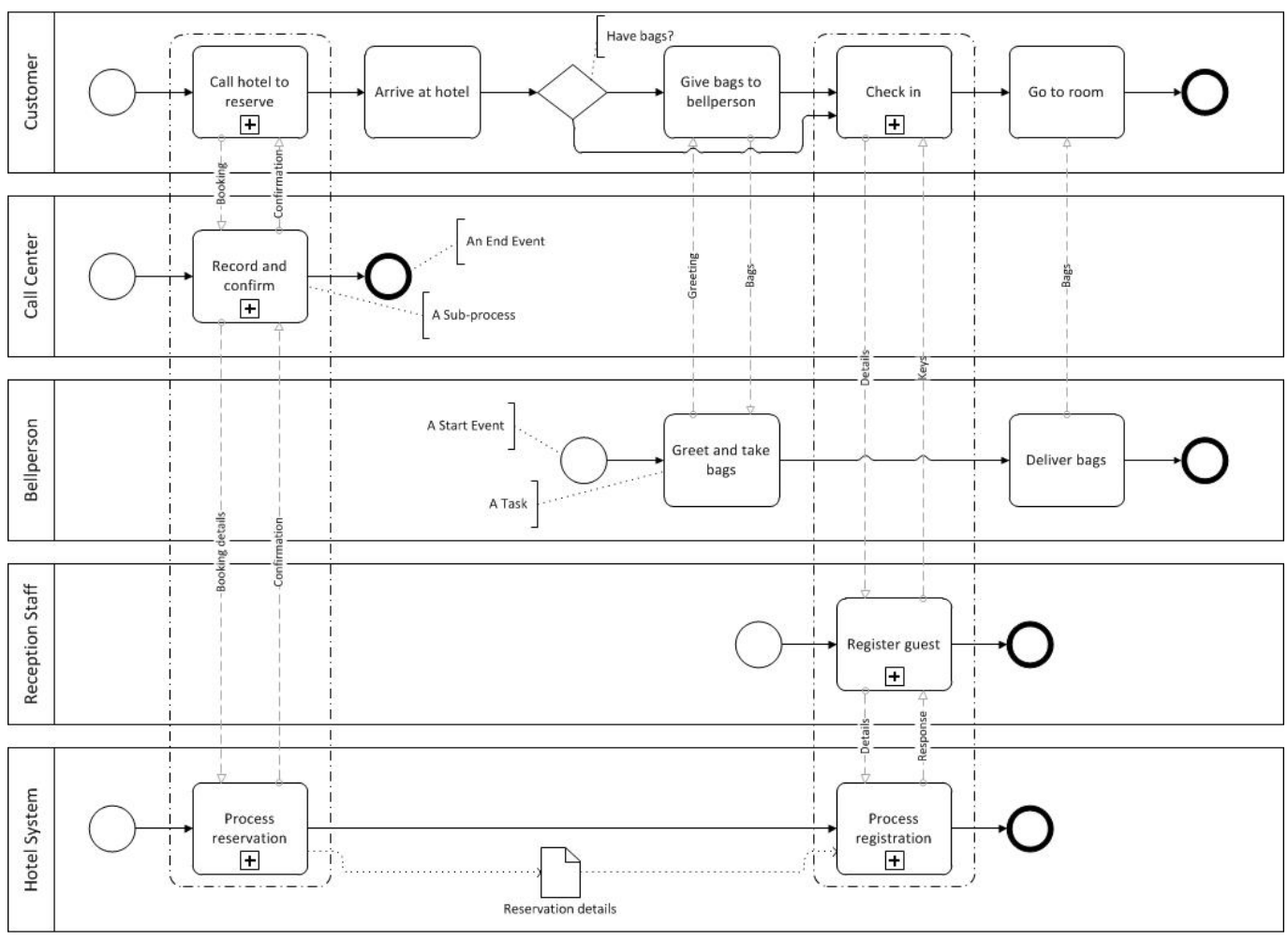

Figure 3. BPMN Diagram example - Hotel Stay

BPMN defines two formal grouping concepts: pool and lane. Pool represents a participant group. These can be a partner (e.g., a company) or a specific partner role (e.g., a buyer, seller, or manufacturer) or group internal to the company (OMG, 2011). Lane is used to categorize activities within a pool. A lane can represent an internal role, an internal department, or system. A process designer may use pool and lane constructs in very different ways and discipline in their respective use is not tight (Kazemzadeh et al., 2015b). In addition, it is critical to know that sequence flows connect activities within a pool and message flows connect activities between different pools.

An association connects BPMN artefacts with flow objects, and is shown with dotted lines, an arrow showing the direction of an association. A data object shows data required for, or produced by, an activity with an association linking data objects to activities.

A group links logically related activities for analysis or documentation purposes, and is shown by a dashed-dotted box around the activities. In the hotel stay example, two sets of activities are grouped: reservation sub-processes and registration sub-processes.

A text annotation allows comments to be included for readers. In the hotel stay example, annotations show different representational symbols for tasks, sub-processes, start events, and end events. Table 3 presents the core concepts of BPMN and their definitions. The next section presents the conceptual evaluation of BPMN against service blueprinting.

\section{Conceptual Evaluation of BPMN against Service Blueprinting}

Table 4 shows the results of the conceptual evaluation of BPMN against service blueprinting. The table indicates the service blueprinting concepts of $<$ Action $>$, $<$ Actor Categories $>$, $<$ Action Flow $>$, $<$ Communication Flow $>$, $<$ Line of Interaction $>,<$ Line of Visibility $>,<$ Line of Internal Interaction $>$ and $<$ Line of Implementation $>$ are completely supported by different BPMN supportive concepts. On the other hand, BPMN does not support $<$ Line of Order Penetration $>$ and $<$ Props and Physical Evidence $>$ in service blueprinting. The following paragraphs utilize semiotic theories to discuss how supportive BPMN concepts cover (or do not cover) each service blueprinting concept according to the represented order of concepts in Table 4.

An $<$ Action $>$ in a service blueprint is an actor's work in a service process. The actor can be a customer, frontline employees (or systems), support, or management. As explained in the BPMN section, an <Activity $>$ "is a 
generic term for work that an organization performs in a process" (OMG, 2011, p.29). This study considers "an organization" in the above definition as a customer or an internal department, system or role of a provider. Therefore, BPMN <Activity $>$ fully supports $<$ Action $>$ in service blueprinting.

Table 3. Concepts in BPMN

\begin{tabular}{|c|c|}
\hline Concept & Definition \\
\hline$<$ Activity $>$ & $\begin{array}{l}\text { An Activity is a generic term for work that an organization performs in a process. An } \\
\text { activity can be atomic or compound. An atomic activity (task) is used when the work in the } \\
\text { process is not broken down to a finer level of detail. A compound activity (sub-process) } \\
\text { comprising more compound activities or tasks. }\end{array}$ \\
\hline$<$ Event $>$ & $\begin{array}{l}\text { An Event is something that "happens" during the course of a Process. These Events affect } \\
\text { the flow of the model and usually have a cause (trigger) or an impact (result). An event can } \\
\text { happen at the start of a process (start event), during a process (intermediate event), or at the } \\
\text { end of a process (end event). }\end{array}$ \\
\hline$<$ Pool $>$ & $\begin{array}{l}\text { A Pool represents a Participant in a process. A Pool is a graphical container for partitioning } \\
\text { a set of Activities from other Pools/participants. }\end{array}$ \\
\hline$<$ Lane $>$ & $\begin{array}{l}\text { A Lane is a sub-partition within a Pool. Lanes are often used for such things as internal } \\
\text { roles (e.g., Manager, Associate), systems (e.g., an enterprise application), or an internal } \\
\text { department (e.g., shipping, finance). }\end{array}$ \\
\hline$<$ Sequence Flow $>$ & $\begin{array}{l}\text { A Sequence flow is used to show the order that activities will be performed in a process by } \\
\text { a participant. Sequence flows are connecting and ordering activities within a pool. }\end{array}$ \\
\hline$<$ Message Flow $>$ & $\begin{array}{l}\text { A Message Flow is used to show the flow of Messages between two Participants that are } \\
\text { prepared to send and receive them. In BPMN, two separate Pools in a Diagram represent } \\
\text { the two Participants. }\end{array}$ \\
\hline$<$ Message $>$ & A Message is used to depict the contents of a communication between two participants. \\
\hline$<$ Gateway $>$ & $\begin{array}{l}\text { A Gateway is used to control the divergence and convergence of Sequence Flows in a } \\
\text { Process. Thus, it will determine traditional decisions, as well as the forking, merging, and } \\
\text { joining of paths. }\end{array}$ \\
\hline$<$ Data Object $>$ & $\begin{array}{l}\text { Data Objects provide information about what Activities require to be performed and/or } \\
\text { what they produce. }\end{array}$ \\
\hline$<$ Text Annotation $>$ & $\begin{array}{l}\text { Text Annotations are a mechanism for a modeler to provide additional information for the } \\
\text { reader of a BPMN Diagram. }\end{array}$ \\
\hline$<$ Association $>$ & $\begin{array}{l}\text { An Association is used to link artefacts (data objects and annotations) to flow objects } \\
\text { (activities, events and gateways). }\end{array}$ \\
\hline$<$ Group $>$ & $\begin{array}{l}\text { A Group identifies logically related activities and does not affect the sequence flow. The } \\
\text { grouping can be used for documentation or analysis purposes. }\end{array}$ \\
\hline
\end{tabular}

Note: Definitions from OMG (2011)

Table 4. Results of conceptual evaluation of BPMN against service blueprinting

\begin{tabular}{ccc}
\hline Concepts of service blueprinting & Degree of overlap & Supportive concepts of BPMN \\
\hline Action $>$ & $\checkmark$ & $<$ Activity $>$ \\
$<$ Actor Categories $>$ & $\checkmark$ & $<$ Pool plus Lane $>$ \\
$<$ Action Flow $>$ & $\checkmark$ & $<$ Sequence Flow plus Message Flow $>$ \\
$<$ Communication Flow $>$ & $\checkmark$ & $<$ Sequence Flow plus Message Flow $>$ \\
$<$ Line of Interaction $>$ & $\checkmark$ & $<$ Pool plus Lane $>$ \\
$<$ Line of Visibility $>$ & $\checkmark$ & $<$ Pool plus Lane $>$ \\
$<$ Line of Internal Interaction $>$ & $\checkmark$ & $<$ Pool plus Lane $>$ \\
$<$ Line of Order Penetration $>$ & $\mathbf{x}$ & \\
$<$ Line of Implementation $>$ & $\checkmark$ & $<$ Pool plus Lane $>$ \\
$<$ Props and Physical Evidence $>$ & $\mathbf{x}$ & \\
\hline
\end{tabular}


Service blueprinting <Actor Categories $>$ refers to the customer, onstage employees, backstage employees (or systems), support employees (or systems), and management employees (or systems). A $<$ Pool $>$ in BPMN can present customer activities, provider activities or categorize provider activities to different organizational department processes and systems, similar to service blueprinting categorization for the provider actions. $<$ Lane $>$ is used to categorize activities within a $<$ Pool $>$. $<$ Lane $>$ can be used to categorize provider activities similar to service blueprint actors. Therefore, BPMN users can employ only $<$ Pool $>$ or a combination of $<$ Pool $>$ and $<$ Lane $>$ to show all actors in service blueprinting. Consequently, $<$ Pool plus Lane $>$ in BPMN fully covers $<$ Actor Categories $>$ in PCN.

$<$ Action Flow $>$ in a service blueprint presents the order of performed actions by an actor. A $<$ Communication Flow $>$ in a service blueprint represents the flow of communication between two actions performed by two different actors. A $<$ Sequence Flow $>$ in a BPMN diagram orders activities by a participant. $<$ Message Flow $>$ in a BPMN diagram shows the flow of messages between two activities performed by two different participants. As we explained above, a BPMN diagram can use $<$ Pool $>$ to represent customer activities and divide provider activities to onstage, backstage, support, and management processes. In this case, a $<$ Sequence Flow $>$ presents the order of activities performed by an actor similar to $<$ Actor Categories $>$ in service blueprinting. Moreover, $<$ Communication Flow $>$ concepts connect two activities performed by two different actors similar to $<$ Actor Categories $>$ in a service blueprint. However, BPMN can categorize provider activities using a combination of $<$ Pool $>$ and $<$ Lane $>$ concepts. Therefore, $<$ Sequence Flow plus Message Flow $>$ in BPMN fully supports $<$ Action Flow $>$ and $<$ Communication Flow $>$ in PCN.

As we discussed in section 3, service blueprinting identifies five lines. The first line is the $<$ Line of Interaction $>$ which is an interface between customer actions and frontline actions. The second line is the <Line of Visibility $>$, which separates onstage actions from backstage actions. The third line is the $<$ Line of Internal Interaction $>$ which divides backstage actions from support processes. The fourth line is the $<$ Line of Order Penetration $>$. This line was introduced by Fließ \& Kleinaltenkamp (2004) to separate customer induced actions from customer independent ones. The last line is the $<$ Line of Implementation $>$, which is an interface between support actions and management processes. As previously discussed, in BPMN only $<$ Pool $>$ or a combination of $<$ Pool $>$ and $<$ Lane $>$ can be used to represent different actors similar to a service blueprint. Therefore, BPMN $<$ Pool plus Lane $>$ fully covers all service blueprinting lines except the $<$ Line of Order Penetration $>$. BPMN cannot support the $<$ Line of Order Penetration $>$ because it does not include any concepts to distinguish provider activities, which involve interaction with customers or customers' resources from activities done independently by a provider.

Finally, BPMN does not represent physical evidence customers can see during service delivery. Service blueprinting $<$ Props and Physical Evidence $>$ includes the tangibles that customers can see and affects customers' perception of service quality. Therefore, there is no concept from BPMN to cover $<$ Props and Physical Evidence $>$ from service blueprinting.

Summarizing, eight out of ten service blueprinting concepts are fully covered by different BPMN concepts. These service blueprinting concepts are: <Action $>$, <Actor Categories $>$, <Action Flow $>,<$ Communication Flow $>,<$ Line of Interaction>, $<$ Line of Visibility $>,<$ Line of Internal Interaction $>$ and $<$ Line of Implementation>. The only two service blueprinting concepts which are not covered by any concepts from BPMN are $<$ Line of Order Penetration $>$ and $<$ Props and Physical Evidence $>$. BPMN does not clarify if a provider activity involves interaction with customers or customers' resources. Moreover, BPMN does not consider customer experience.

The following section details the results of conceptual evaluation of service blueprinting against BPMN in a tabular form and discusses them in detail.

\section{Conceptual Evaluation of Service Blueprinting against BPMN}

Table 5 shows the results of the conceptual evaluation of service blueprinting against BPMN. The table shows there are three concepts from BPMN which are completely supported by service blueprint supportive concepts: $<$ Activity $>$, $<$ Sequence Flow $>$ and $<$ Message Flow $>$. In addition to these, $<$ Lane $>$ and $<$ Pool $>$ concepts are partially covered by service blueprinting. The evaluation results indicate no coverage for $<$ Event $>,<$ Gateway $>$, $<$ Message $>$, $<$ Data Object $>$, <Text Annotation $>$, $<$ Association Flow $>$ and $<$ Group $>$ by any concepts from service blueprinting. The following paragraphs discuss the nature of the gaps according to the table order.

An $<$ Activity $>$ in a BPMN diagram refers to work that someone does as part of a process. An $<$ Action $>$ in a service blueprint is defined as the work done by an actor. Therefore, an $<$ Action $>$ in a service blueprint can refer to the $<$ Action $>$ concept performed by customers, frontline employees (or systems), or if they are a part of the 
support and management processes. As mentioned in section 4, OMG (2011, p.29) identifies two types of BPMN $<$ Activity $>$ : atomic and compound. An atomic $<$ Activity $>$ (task) is a part of a service process which cannot be broken down to finer levels of detail. A compound $<$ Activity $>$, also called a sub-process, consists of more than one compound $<$ Activity $>$ or task. In comparison, an $<$ Action $>$ in service blueprinting can also be a task or is possible to be broken down into more than one $<$ Action $>$. As a result, $<$ Action $>$ in service blueprinting fully supports $<$ Activity $>$ in PCN.

Table 5. Results of conceptual evaluation of service blueprinting against BPMN

\begin{tabular}{ccc}
\hline Concepts of BPMN & Degree of overlap & Supportive concepts of service blueprinting \\
\hline$<$ Activity $>$ & $\checkmark$ & $<$ Action $>$ \\
$<$ Event $>$ & $\mathbf{x}$ & $<$ Actor Categories $>$ \\
$<$ Pool $>$ & $\checkmark \mathrm{p}$ & $<$ Actor Categories $>$ \\
$<$ Lane $>$ & $\checkmark \mathrm{p}$ & $<$ Action Flow plus Communication Flow $>$ \\
$<$ Sequence Flow $>$ & $\checkmark$ & $<$ Action Flow plus Communication Flow $>$ \\
$<$ Message Flow $>$ & $\checkmark$ & \\
$<$ Message $>$ & $\mathbf{x}$ & \\
$<$ Gateway $>$ & $\mathbf{x}$ & \\
$<$ Data Object $>$ & $\mathbf{x}$ & \\
$<$ Text Annotation $>$ & $\mathbf{x}$ & \\
$<$ Association Flow $>$ & $\mathbf{x}$ & \\
$<$ Group $>$ & & \\
\hline
\end{tabular}

OMG (2011, p. 29) defines <Event> as "something that happens during the course of a Process. These Events affect the flow of the model and usually have a cause (trigger) or an impact (result). There are three types of Events, based on when they affect the flow: Start, Intermediate, and End". Considering this definition for an $<$ Event $>$ in BPMN, there is no concept in PCN to cover it.

A $<$ Pool $>$ represents a participant that is involved in a service process. A $<$ Lane $>$ organizes or categorizes provider activities based on internal departments, roles or systems and is a part of a $<$ Pool $>$. The comparison of concepts in service blueprinting with $<$ Lane $>$ and $<$ Pool $>$ shows that service blueprinting $<$ Actor Categories $>$ partially covers them. Service blueprinting $<$ Actor Categories $>$ divides actions in a service process into five different categories: customer actions, onstage actions, backstage actions, support and management processes. This is similar to categorizing actors involved in a service process, using only $<$ Pool $>$, or a combination of $<$ Pool $>$ and $<$ Lane $>$. In fact, $<$ Pool $>$ and $<$ Lane $>$ can group activities of a service process in many different ways. Therefore, service blueprinting $<$ Actor Categories $>$ is a specific way of categorizing actions in a BPMN diagram using $<$ Pool $>$ and $<$ Lane $>$, so it covers them partially.

BPMN $<$ Sequence Flow $>$ orders the activities done by a participant in a service process. $<$ Message Flow $>$ is defined by OMG (2011) as the connection between two participants, one a sender and the other one a receiver. In a service blueprint, $<$ Action Flow $>$ orders a specific actor's actions and $<$ Communication Flow $>$ represents communication between two actors. An actor's actions in a service blueprinting can be similar to a participant's activities or a combination of two or more participants' activities in BPMN. Therefore, <Action Flow plus Communication Flow $>$ fully covers $<$ Sequence Flow $>$ and $<$ Message Flow $>$.

Every $<$ Message Flow $>$ has a $<$ Message $>$. A $<$ Message $>$ presents the content of $<$ Message Flow $>$ and shows what an actor sends to another actor. Service blueprint does not cover $<$ Message $>$ because it does not show the content of communications between two different actors.

A $<$ Gateway $>$ controls the divergence or convergence of a $<$ Sequence Flow $>$ in a service process. A $<$ Gateway $>$ determines branching, forking, merging, or joining of paths through the use of an internal condition. Service blueprinting does not include any concepts to change the order of actions based on a specific condition. The study of several blueprints implies that service blueprinting modelers always avoid any conditions to order actions; they restrict the diagram to one possible condition. Therefore, $<$ Gateway $>$ in BPMN is not covered by any concepts from service blueprinting.

A $<$ Data Object $>$ is defined as required data for an activity or produced data by an activity. It is essential to know that "data input and data output provide the same information for processes" (OMG, 2011). Recker (2011) 
explains that $<$ Data Object $>$ is a kind of artefact; it provides information about processed data and does not have any direct effect on connecting objects. Service blueprint does not show if there is a need for data to perform an activity or produced data during an activity.

A < Text Annotation $>$ is an artefact in BPMN diagrams, providing additional information about different concepts. In addition, the literature suggests that $<$ Text Annotation $>$ is employed in many cases to represent business rules (Recker, Indulska, Rosemann, \& Green, 2010). In comparison, it seems service marketers assumed everything was clear in a service blueprint and that there was no need to add additional information for readers.

An $<$ Association $>$ is used to associate information with flow objects. An $<$ Association $>$ can be used especially when business analysts want to associate a $<$ Text Annotation $>$ or a $<$ Data Object $>$ to other represented concepts (e.g. events and activities). Service blueprinting concepts do not include any tools to associate information with activities. Consequently, $<$ Association $>$ in BPMN is not covered by service blueprinting.

$<$ Group $>$ is a concept identifying a category of activities for the purpose of process analysis and documentation without affecting the order of activities. When the designers use a $<$ Group $>$, they draw a rounded corner box with dashed lines around targeted graphical elements and they name it with a label. BPMN designers and analysts can use a $<$ Group $>$ to categorize activities within a pool or across pools (Recker, 2011). Service Blueprint users can only categorize actions based on their actor. Therefore, Service blueprint does not include any concepts that cover $<$ Group $>$ in BPMN.

In summary, service blueprinting fully or partially covers five of twelve BPMN concepts. The fully covered BPMN concepts by service blueprinting are: $<$ Activity $>,<$ Sequence Flow $>$ and $<$ Message Flow $>$. However, $<$ Message $>$ which indicates the content of communication between two participants in a BPMN diagram is not covered at all by any concepts from service blueprinting. In addition to these, $<$ Pool $>$ and $<$ Lane $>$ are partially supported by $<$ Actor Categories $>$ in service blueprinting. Service blueprinting does not cover the basic BPMN flow objects of $<$ Event $>$ and $<$ Gateway $>$. Finally, $<$ Association Flow $>$ and BPMN artefacts including $<$ Data Object $>,<$ Text Annotation $>$, and $<$ Group $>$ are not covered by any service blueprinting concepts.

The following sections present major findings from both comparisons and their conclusions.

\section{Findings}

The results indicate that $<$ Action $>$ from service blueprinting covers the same semantic field as $<$ Activity $>$ in BPMN. Recker (2011) highlights $<$ Activity $>$, $<$ Event $>$, and $<$ Gateway $>$ as the most basic concepts for designing a BPMN diagram. $<$ Event $>$ and $<$ Gateway $>$ are not covered by any concepts from service blueprinting.

The conceptual evaluation of BPMN against Service blueprinting implies that $<$ Sequence Flow plus Message Flow $>$ fully supports $<$ Action Flow $>$ and $<$ Communication Flow $>$. On the other hand, the outcome of conceptual evaluation of service blueprinting against BPMN indicates that $<$ Action Flow plus Communication Flow $>$ fully supports $<$ Sequence Flow $>$ and $<$ Message Flow $>$. Consequently, <Action Flow plus Communication Flow $>$ covers the same semantic field as $<$ Sequence Flow plus Message Flow $>$.

We found that $<$ Pool plus Lane $>$ in BPMN covers a broader semantic field than $<$ Actor Categories $>$ and traditional lines of service blueprinting. This fact results in complete coverage of $<$ Actor Categories $>,<$ Line of Interaction $>,<$ Line of Visibility $>,<$ Line of Internal Interaction $>$, and $<$ Line of Implementation $>$ from service blueprinting by $<$ Pool plus Lane $>$ from BPMN. However, $<$ Line of Order Penetration $>$ is not covered by any concepts from BPMN. The reason is that while the other lines in service blueprinting separate different actors, the $<$ Line of Order Penetration $>$ considers the involvement of participant entities in performed actions and separates actions to two groups; customer induced and customer independent. This is not the way that $<$ Pool $>$ and $<$ Lane $>$ in BPMN categorize activities. $<$ Pool $>$ and $<$ Lane $>$ can categorize activities in a service process to different participant entities, internal departments, roles, and systems.

Another major finding suggests that BPMN does not provide any concepts regarding customer experience. While the first part of a service blueprint diagram presents $<$ Props and Physical Evidence $>$, which involves all evidence visible to customer during service process, BPMN lacks any similar concept. Consequently, a BPMN diagram presents less customer specific perspective. In contrast, customer experience and the interaction of customer with the provider is a key issue for service process designers and service process marketers who employ the service blueprinting method to present service delivery.

Finally, it is understood that not all of the artefacts in BPMN are covered by service blueprint concepts. BPMN defines $<$ Data object $>$ to represent the input and output data of activities. Service blueprinting does not provide any information about involved data in a service process. This difference between BPMN and blueprinting 
implies that BPMN has an informational view, while service blueprinting does not consider any informational aspects of service processes.

$<$ Text Annotation $>$ is purely used to add information about flow objects in a BPMN diagram. $<$ Group $>$ is used to categorize activities for the purpose of service process documentation. Service blueprinting does not provide any concepts to add additional information to the diagrams. As a result, using these artefacts enable BPMN diagram designers to add extra information about represented concepts and reduce further confusion of service process readers and analysts. These all contribute to a better understanding of service processes presented in BPMN diagrams in comparison to service blueprints.

\section{Conclusion}

In conclusion, having $<$ Pool $>$ and $<$ Lane $>$ concepts in BPMN enables BPMN users to categorize the activities in many different intended ways including similar ways to $<$ Actor Categories $>$ in service blueprinting. Therefore, BPMN users can depict visible and invisible actions of a customer. Moreover, $<$ Data Object $>$ in BPMN represents data input and output of activities and illustrates an information view of service processes. All of this implies that BPMN is a better process modeling formalism for designing digitized services compared to service blueprinting, which can be used for process simulation and automation.

It is possible also, in a BPMN diagram, to add additional explanations for different represented concepts and to group a set of activities for the purpose of process documentation and analysis. In comparison, Service Blueprinting has a higher capacity to represent user experience and is a suitable process modeling formalism to design a customer-provider interface.

Service blueprinting is an appropriate method when a company has a customer-centric view toward its service delivery process. A company can utilize service blueprints to integrate a customer focus across the organization and enable customer-oriented business practices. Many companies have successfully used service blueprinting to increase customer satisfaction and repeat business.

BPMN can be used when the practitioners aim to depict organizational departments, systems, and roles that are involved in service delivery process in detail. BPMN has the capability to represent any parts of the service provider organization and their interaction with customers. Inter-organizational communications in a BPMN diagram can also be represented very well. Organizational and informational views of BPMN make it a preferable modeling approach for information systems analysts and professionals.

\section{Acknowledgments}

The authors would like to thank Mr. Christopher Kessaris for the editing of this paper.

\section{References}

Alonzo-Helton, M., Fletcher-Brown, T., \& Stephens, N. (2013). Service Blueprinting. Parking Professional, 29(1), 26-29.

Bitner, M. J., Ostrom, A. L., \& Morgan, F. N. (2008). Service blueprinting: a practical technique for service innovation. California Management Review, 50(3), 66-94.

BPMI.org. (2004). Business Process Modeling Notation (BPMN).

BPMI.org, \& OMG. (2006). Business process modeling notation (BPMN) specification: final adopted specification.

Chinosi, M., \& Trombetta, A. (2012). BPMN: an introduction to the standard. Computer Standards \& Interfaces, 34(1), 124-134. http://dx.doi.org/10.1016/j.csi.2011.06.002

Fließ, S., \& Kleinaltenkamp, M. (2004). Blueprinting the service company. Journal of Business Research, 57(4), 392-404. http://dx.doi.org/10.1016/S0148-2963(02)00273-4

Kazemzadeh, Y., Milton, S. K., \& Johnson, L. W. (2015a). A comparison of concepts in service blueprinting and process-chain-network (PCN). International Journal of Business and Management, 10(4), 13-25. http://dx.doi.org/10.5539/ijbm.v10n4p13

Kazemzadeh, Y., Milton, S. K., \& Johnson, L. W. (2015b). An explication of three service business process modeling approaches. Australian Journal of Business and Economic Studies, 1(2), 40-53.

Kazemzadeh, Y., Milton, S. K., \& Johnson, L. W. (2015c). Process chain Network (PCN) and business process modeling notation (BPMN): a comparison of concepts. Journal of Management and Strategy, 6(1), 88-99. http://dx.doi.org/10.5430/jms.v6n1p88 
Kazemzadeh, Y., Milton, S. K., \& Johnson, L. W. (in press). Service blueprinting and process-chain-network (PCN): an ontological comparison. International Journal of Qualitative Research in Services.

Kingman-Brundage, J. (1989). The ABCs of service system blueprinting. In Designing a winning service strategy (pp. 30-33).

Kingman-brundage, J. (1991). Technology, Design and Service Quality. International Journal of Service Industry Management, 2(3), 47-59.

Kingman-brundage, J. (1993). Service mapping: gaining a concrete perspective on service system design. In The service quality handbook (pp. 148-163).

Kingman-brundage, J. (1995). Service mapping: back to basics. In Understanding services management (pp. 119-142).

Ko, R. K. L., Lee, S. S. G., \& Lee, E. W. (2009). Business process management (BPM) standards: a survey. Business Process Management Journal, 15(5), 744--791. http://dx.doi.org/10.1108/14637150910987937

Milton, S. K., \& Johnson, L. W. (2012). Service blueprinting and BPMN: a comparison. Managing Service Quality, 22(6), 606-621. http://dx.doi.org/10.1108/09604521211287570

Milton, S. K., \& Kazmierczak, E. (2004). An ontology of data modelling languages. Journal of Database Management, 15(2), 19-38.

Muehlen, M., \& Recker, J. (2008). How Much Language is Enough? Theoretical and Practical Use of the Business Process Modeling Notation. In Proceedings of 20th International Conference on Advanced Information Systems Engineering (pp. 1-15). Montpellier, France.

Ojasalo, J. (2012). Constrasting theoretical grounds of business process modelling and service blueprinting. In Proceedings of the 7th Global Conference on Business and Finance (Vol. 7, pp. 410-420). San Jose, Costa Rica.

OMG. (2011). Business Process Model and Notation (BPMN).

Recker, J. (2010). Opportunities and constraints: the current struggle with BPMN. Business Process Management Journal, 16(1), 181-201. http://dx.doi.org/10.1108/14637151011018001

Recker, J. (2011). Evaluations of process modeling grammars. In Lecture Notes in Business Information Processing. Springer-Verlag Berlin Heidelberg.

Recker, J., Indulska, M., Rosemann, M., \& Green, P. (2010). The ontological deficiencies of process modeling in practice. European Journal of Information Systems, 19(5), 501-525. http://dx.doi.org/10.1057/ejis.2010.38

Shostack, G. L. (1982). How to design a service. European Journal of Marketing, 16(1), 49-63.

Shostack, G. L. (1984). Designing services that deliver. Harvard Business Review, 62(1), 133-139.

Shostack, G. L. (1987). Service positioning through structural change. Journal of Marketing, 51(1), 34-43.

\section{Copyrights}

Copyright for this article is retained by the author(s), with first publication rights granted to the journal.

This is an open-access article distributed under the terms and conditions of the Creative Commons Attribution license (http://creativecommons.org/licenses/by/3.0/). 